Federal Reserve Bank of New York

Staff Reports

\title{
Does Central Clearing Reduce Counterparty Risk in Realistic Financial Networks?
}

\author{
Rodney Garratt \\ Peter Zimmerman
}

Staff Report No. 717

March 2015

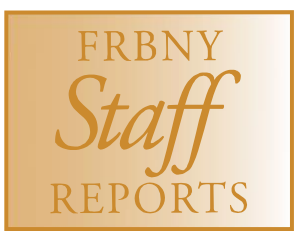

This paper presents preliminary findings and is being distributed to economists and other interested readers solely to stimulate discussion and elicit comments. The views expressed in this paper are those of the authors and do not necessarily reflect the position of the Federal Reserve Bank of New York or the Federal Reserve System. Any errors or omissions are the responsibility of the authors. 


\author{
Does Central Clearing Reduce Counterparty Risk in Realistic Financial Networks? \\ Rodney Garratt and Peter Zimmerman \\ Federal Reserve Bank of New York Staff Reports, no. 717 \\ March 2015 \\ JEL classification: D85, G14
}

\begin{abstract}
Novating a single asset class to a central counterparty $(\mathrm{CCP})$ in an over-the-counter derivatives trading network impacts both the mean and variance of total net exposures between

counterparties. When a small number of dealers trade in a relatively large number of asset classes, central clearing increases the mean and variance of net exposures, which may lead to increased counterparty risk and higher margin needs. There are intermediate cases where there is a tradeoff: The introduction of a CCP leads to an increase in expected net exposures but this increase is accompanied by a reduction in variance. We extend the work of Duffie and Zhu (2011) by considering general classes of network structures and focus on scale-free and core-periphery structures, which have been shown to be accurate models of real-world financial networks. We find that a CCP is unlikely to be beneficial when the link structure of the network relies on just a few key nodes. In particular, in large scale-free networks a CCP will always worsen expected netting efficiency. In such cases, CCPs can improve netting efficiency only if agents have some degree of risk aversion that allows them to trade off the reduced variance against the higher expected netted exposures. This may explain why, in the absence of regulation, traders in a derivatives network may not develop a CCP themselves.
\end{abstract}

Key words: central clearing, core-periphery network, scale-free network

Garratt: Federal Reserve Bank of New York (e-mail: rodney.garratt@ny.frb.org).Zimmerman: Saïd Business School, University of Oxford (e-mail: peter.zimmerman@sbs.ox.ac.uk). This paper is based on work initiated when both authors were at the Bank of England. The authors wish to thank Marco Galbiati, Christopher Lewis, Lavan Mahadeva, Mark Manning, and seminar participants at the Bank of England, the Center for Latin American Monetary Studies, the International Banking, Economics, and Finance Association / Allied Social Science Association, and the Saïd Business School for useful comments and suggestions. The views expressed in this paper are those of the authors and do not necessarily reflect the positions of the Bank of England, the Federal Reserve Bank of New York, or the Federal Reserve System. 


\section{Introduction}

One of the benefits of central clearing is that it reduces the aggregate level of exposures in the system by netting offsetting claims, which can reduce systemic risk and the amount of margin required by counterparties. This is true in a world where all asset classes are simultaneously novated to a central counterparty (CCP). However, Duffie and Zhu (2011) show that, when a single asset class is switched to central clearing, bilateral exposures between dealers may increase - as a result of reduced netting opportunities across pairs of dealers - resulting in an overall loss in netting efficiency.

Duffie and Zhu's results are not based on actual exposure networks. Rather they express the total exposure of one dealer to another of all positions in each asset class by a random variable. They specify a distribution that is assumed to generate these exposures and their results are based on expected exposures with respect to this distribution. Because they assume homogeneity in the distributions across all dealers, they can compute the average total exposure with or without a CCP by looking at a single dealer. In short, all dealers are ex ante the same in their analysis. Taking as given the distribution used to generate exposures, this allows them to express conditions for whether or not a CCP is beneficial solely in terms of the number of asset classes and the number of dealers.

The clear advantage of this approach is that it is not necessary to observe the actual network exposures; instead it relates the question of whether or not the introduction of a CCP in a single asset class is beneficial or not to easily observable parameters. However, a downside of this approach is that the implied networks are too homogeneous, which means they may not be realistic. Typically real-world financial networks have a number of well-connected counterparties coupled with a larger number of more poorly-connected counterparties. These features have been shown to be well-explained by scale-free network models (e.g. Soramäki et al (2007), Inaoka et al (2004), Garlaschelli et al (2005)) and by core-periphery models (e.g. Craig and von Peter (2014), Langfield, Liu and Ota (2014), Markose (2012)).

We extend the results of Duffie and Zhu so that they can be applied to any network. And, in addition to examining the effect of introducing a CCP on the expected net exposures of dealers, we also explore the effect on the variance of these net exposures. The rationale for this is that risk-averse dealer agents may wish to minimise the variance as well as the expectation of their net exposures.

We find that, when the number of asset classes is small relative to the number of dealers, introducing a CCP is likely to reduce both the mean and variance of net exposures. But a CCP is less likely to be beneficial for networks where a high proportion of the links rely on a few key nodes. In some intermediate cases, introducing a CCP may have opposite effects on the mean and variance of netted exposures, leading to a trade-off. The optimal policy then is likely to depend on the degree of risk-aversion among counterparties and, potentially, regulators.

Both scale-free and core-periphery structures can be generated by simple and intuitive processes. These incorporate the growing structure of the network and preferential attachment: that is, new nodes are likely to attach themselves to nodes which are larger or more successful. In the case of scale-free networks, new nodes are more likely to attach themselves to nodes with a large number of existing connections (Barabási and 
Albert (1999)). And core-periphery networks may emerge if there is heterogeneity between nodes, with links to a particular class of nodes being more attractive (e.g. Van der Leij, in't Veld and Hommes (2014)).

We argue that this approach provides an appropriate technique for generating the unobserved link-structure of the network, while preserving the ex ante symmetry assumptions that are necessary to obtain analytic results. Generating scale-free networks using the specific network formation process introduced by Dorogovtsev, Mendes and Samukhin (2001), we find that expected net exposures always increase when a single asset is novated to a CCP, regardless of the size of the network. However, for risk-averse agents the cost of this reduction in netting efficiency may be offset by the benefit of a reduction in variance. This may be an important justification for regulatory intervention to introduce a CCP, if the dealer agents are less risk-averse than would be socially optimal. For smaller core-periphery networks our findings are similar, but there is a critical size beyond which introducing a CCP is unambiguously good for netting efficiency in such networks.

One application of our paper is on the impact on margin requirements of introducing a CCP. Sidanius and Žikeš (2012) explain the issue and estimate the impact, while more recent papers including Duffie, Scheicher and Vuillemey (2014) and Campbell (2014) use actual bilateral exposure data to analyze the issue empirically.

\section{A review of the relevant literature}

\subsection{Articles which follow the framework of Duffie and Zhu (2011)}

The framework developed by Duffie and Zhu (2011) has been utilized and developed by other authors in order to investigate specific problems. Heath, Kelly and Manning (2013) is perhaps the most similar to our paper in that they assume a core-periphery structure for the network, rather than maintaining Duffie and Zhu's assumptions that all dealers are ex ante the same. They then use a computational approach to compare the efficiency of various clearing arrangements.

Anderson, Dion and Pérez Saiz (2013) and Cox, Garvin and Kelly (2013) apply the Duffie and Zhu framework to explore the policy issue of interoperability between CCPs. They use the model to examine whether a regulator can reduce netted exposures by mandating trades to be novated to a local CCP, which can link to a global CCP that clears a range of products. Both papers retain the assumption of a homogeneous link network, though Cox, Garvin and Kelly allow for some heterogeneity between dealer agents in the magnitude of exposures (but not the existence of links).

Cont and Kokholm (2014) extend the Duffie and Zhu framework by relaxing the assumption of normal exposures between counterparties and show using a simulation approach that Duffie and Zhu's conclusions are potentially sensitive to different distributional assumptions. However, they retain the homogeneous network assumption that Duffie and Zhu use. This is in contrast to our paper, which uses more general and realistic network structures. While our framework explicitly permits the use of non-normal exposures, some distributional assumption is necessary in order to obtain analytical results. 
Our paper makes two key innovations to the Duffie and Zhu model which, to our knowledge, have not been considered before. First, we look at how the introduction of a CCP affects the variance, as well as the mean, of net exposures. Second, we provide an analytical generalization of the model so that it can be applied to any network.

\subsection{Other articles on network models of CCPs}

There are a variety of papers which use a network approach to examine the contagion dynamics of OTC derivatives networks, rather than netting efficiency. Markose (2012) find that the empirical OTC derivatives network aggregated over all products can be well-described by a modified core-periphery model, and derive summary statistics to identify institutions which carry the greatest quantity of systemic risk. Borovkova and Lalaoui El Mouttalibi (2013) use a simulation approach to model the effect of the introduction of a CCP on default cascades in a network. They examine both homogeneous (Erdős-Rényi) and core-periphery networks, and find that homogeneous networks are more resilient.

Jackson and Manning (2007) and Galbiati and Soramäki (2012) use different approaches to examine the desirability of tiering — that is, restricting direct access to the $\mathrm{CCP}$ to a limited set of counterparties. Jackson and Manning examine the impact using a range of metrics, while Galbiati and Soramäki focus on the impact on exposures measured as averages over time, or across direct counterparties, in terms of a single asset class. Song, Sowers and Jones (2014) extend the Galbiati and Soramäki framework to study the effect of network structure on the maximum exposure risk of the CCP itself, and use extreme value theory to obtain analytical results.

\section{A general network model of exposure netting}

We assume that the dealer network is not directly observable but the number of nodes, their degree distribution and the distribution of the magnitude of bilateral exposures is known. This is a realistic assumption for dealer networks, where the regulator and participants often lack exact real-time knowledge of bilateral exposures. This is a generalization of the assumption made in Duffie and Zhu (2011), where the exact structure of the network is known. Our framework builds on and extends theirs.

Let $N$ be the number of nodes (i.e. market participants) and let $S$ be a random variable denoting the number of links a given node has. Links are undirected. Define $J_{i}$ to be the set of nodes with which node $i$ has a link. For each $i$, the size of this set is determined by a realization of the random variable $S$.

Let $K$ denote the number of asset classes. Define $X_{i j}$ to be a $K$-vector of values (weights) on the link between nodes $i$ and $j$. The $k^{\text {th }}$ element in each vector, denoted $X_{i j}^{k}$, represents the net exposure between the two nodes in asset class $k$. It is positive or negative according to the direction of the net exposure. Each value is generated independently with the same known distribution.

First consider the situation without a CCP. Suppose nodes $i$ and $j$ are linked. Define

$Y_{i j}^{K} \equiv \max \left(\sum_{k=1}^{K} X_{i j}^{k}, 0\right)$ to be the value of note $i$ 's netted exposure to node $j$. Note that positive net exposures in one asset class can be partially or wholly offset by negative 
net exposures in another asset class with the same counterparty. If $i$ and $j$ are not linked, then the net exposure is zero. The total net exposure of node $i$ therefore equals $\sum_{j \in J_{i}} Y_{i j}^{K}$.

Now define the function $f$ as the expected net exposure between any two nodes:

$$
f(K) \equiv \mathrm{E}\left[Y_{i j}^{K}\right]
$$

The expected total netting efficiency for a given node $i$ is:

$$
\begin{aligned}
\phi_{N, K} & \equiv \mathrm{E}\left[\sum_{j \in J_{i}} Y_{i j}^{K}\right] \\
& =\mathrm{E}\left[\mathrm{E}\left[\sum_{j \in J_{i}} Y_{i j}^{K} \mid S\right]\right] \\
& =\mathrm{E}[S f(K)] \\
& =\mathrm{E}[S] f(K),
\end{aligned}
$$

where we have used the fact that each $Y_{i j}^{K}$ is independent from one another, and from $S$.

Similarly, the variance of the exposure between two nodes after netting is:

$$
g(K) \equiv \operatorname{Var}\left[Y_{i j}^{K}\right]
$$

and the variance of the total netting efficiency of the network is:

$$
v_{N, K} \equiv \operatorname{Var}\left[\sum_{j \in J_{i}} Y_{i j}^{K}\right] .
$$

We can evaluate this expression using the law of total variance:

$$
\begin{aligned}
v_{N, K} & =\mathrm{E}\left[\operatorname{Var}\left[\sum_{j \in J_{i}} Y_{i j}^{K} \mid S\right]\right]+\operatorname{Var}\left[\mathrm{E}\left[\sum_{j \in J_{i}} Y_{i j}^{K} \mid S\right]\right] \\
& =\mathrm{E}[S g(K)]+\operatorname{Var}[S f(K)] \\
& =\mathrm{E}[S] g(K)+\operatorname{Var}[S] f(K)^{2} .
\end{aligned}
$$

\subsection{Novating a single asset class to a $\mathrm{CCP}$}

Now we introduce a CCP in a single asset class. Without loss of generality, let the centrally cleared asset class be the one labelled $K$. The net exposure of a given node $i$ becomes:

$$
\sum_{j \in J_{i}} Y_{i j}^{K-1}+\underbrace{\max \left(\sum_{j \in J_{i}} X_{i j}^{K}, 0\right)}_{Y_{i, C C P}^{S}}
$$

where the first term is the sum of a node's exposures to the other nodes, and the second term is its netted exposure to the CCP. We can rewrite the second term as $Y_{i, C C P}^{S}$, with the $S$ superscript arising because the size of $J_{i}$ has distribution $S$. 
Note that, for a given realized value of $S$, the two terms in (6) are independent: the first term is determined entirely by exposures arising from the first $K-1$ assets, while the second is determined entirely by exposures due to asset $K$.

Now, when there is a CCP, the expected total net exposure of node $i$ is:

$$
\begin{aligned}
\widetilde{\phi_{N, K}} & =\mathrm{E}\left[\mathrm{E}\left[\sum_{j \in J_{i}} Y_{i j}^{K-1}+Y_{i, C C P}^{S} \mid S\right]\right] \\
& =\mathrm{E}[S f(K-1)+f(S)] \\
& =\mathrm{E}[S] f(K-1)+\mathrm{E}[f(S)],
\end{aligned}
$$

and the variance of the total net exposure of node $i$ is:

$$
\begin{aligned}
\widetilde{v_{N, K}} & =\mathrm{E}\left[\operatorname{Var}\left[\sum_{j \in J_{i}} Y_{i j}^{K-1}+Y_{i, C C P}^{S} \mid S\right]\right]+\operatorname{Var}\left[\mathrm{E}\left[\sum_{j \in J_{i}} Y_{i j}^{K-1}+Y_{i, C C P}^{S} \mid S\right]\right] \\
& =\mathrm{E}[S g(K-1)+g(S)]+\operatorname{Var}[S f(K-1)+f(S)] \\
& =\mathrm{E}[S] g(K-1)+\mathrm{E}[g(S)]+\operatorname{Var}[S f(K-1)+f(S)] .
\end{aligned}
$$

Using (2) and (7), the change in expected net exposure that results from novating a single asset class to a $\mathrm{CCP}$ is:

$$
\widetilde{\phi_{N, K}}-\phi_{N, K}=\mathrm{E}[S] f(K-1)+\mathrm{E}[f(S)]-\mathrm{E}[S] f(K) .
$$

Using (5) and (8), the change in variance that results from novating a single asset class to a $\mathrm{CCP}$ is:

$$
\begin{aligned}
& \widetilde{v_{N, K}}-v_{N, K}= \\
& \quad \operatorname{Var}[S f(K-1)+f(S)]-\operatorname{Var}[S] f(K)^{2}-\mathrm{E}[S](g(K)-g(K-1))+\mathrm{E}[g(S)] .
\end{aligned}
$$

These results show that introducing a CCP may change both the mean and variance of total net exposures. Assuming some degree of risk aversion, reduction in either the mean or the variance is likely to be positive for users of the system, since it means that counterparty risk - and total margin needs - are lower either in expectation or volatility. Therefore we can say that a CCP brings netting benefits when either (9) or (10) are negative.

Note that in the case $K=1$, the CCP clears all of the asset classes that the dealers trade with one another so we would always expect the introduction of a CCP to improve netting, as it does not disrupt any of the existing bilateral netting sets. This is confirmed by equations (9) and (10), which both have negative right-hand sides when $K=1{ }^{1}$

We assume that the agents in our network prefer to reduce both the expected value and variance of total net exposures. It is quite possible, however, that introducing a CCP may increase the expected value of total net exposures and reduce the variance, or vice versa. In such cases there will be a trade-off between the two effects. Whether this increases overall welfare or not will depend on the utility functions of the agents:

${ }^{1}$ To show this, note that $f(0)=0=g(0)$ and use the fact that the $\max (\cdot, 0)$ function is sub-additive. 
we might expect relatively risk-averse agents to place more weight on the reduction in variance and less weight on the increase in expected exposures. One key focus of this paper is to identify the extent to which the trade-off depends on the underlying network structure.

\subsection{Assigning a distribution to the bilateral exposures}

To obtain tractable results for the cases where $K>1$, we need to assume a distribution for the bilateral exposures between dealers. This will enable us to write down expressions for $f$ and $g$ in equations (9) and (10). We follow Duffie and Zhu (2011) and assume that each of the bilateral exposures $X_{i j}^{k}$ is independent and identically normally distributed with mean 0 and variance $\sigma^{2}$. Using the formula for the sum of independent normal random variables, we can write the function $f$ as:

$$
\begin{aligned}
f(\theta) & =\int_{0}^{\infty} \frac{1}{\sqrt{2 \pi \theta} \sigma} y e^{-\frac{y^{2}}{2 \theta \sigma^{2}}} \mathrm{~d} y \\
& =\sigma \sqrt{\frac{\theta}{2 \pi}}
\end{aligned}
$$

and:

$$
\begin{aligned}
g(\theta) & =\int_{0}^{\infty} \frac{1}{\sqrt{2 \pi \theta} \sigma} y^{2} e^{-\frac{y^{2}}{2 \theta \sigma^{2}}} \mathrm{~d} y-\sigma^{2} \frac{\theta}{2 \pi} \\
& =\frac{1}{2} \int_{-\infty}^{\infty} \frac{1}{\sqrt{2 \pi \theta} \sigma} y^{2} e^{-\frac{y^{2}}{2 \theta \sigma^{2}}} \mathrm{~d} y-\sigma^{2} \frac{\theta}{2 \pi} \\
& =\sigma^{2}(\pi-1) \frac{\theta}{2 \pi} .
\end{aligned}
$$

We substitute these into (9) and (10) to show that a CCP reduces the expectation of net exposures if and only if:

$$
\sqrt{K}+\sqrt{K-1}<\frac{\mathrm{E}[S]}{\mathrm{E}[\sqrt{S}]},
$$

and introducing a CCP reduces the variance of net exposures if and only if:

$$
\operatorname{Var}[S \sqrt{K-1}+\sqrt{S}]<K \operatorname{Var}[S] .
$$

Rewriting the first variance term in (13) in terms of expectations operators, we can express this condition as:

$$
2 \sqrt{K-1}<\frac{\operatorname{Var}[S]-\operatorname{Var}[\sqrt{S}]}{\mathrm{E}\left[S^{\frac{3}{2}}\right]-\mathrm{E}[S] \mathrm{E}[\sqrt{S}]}
$$

where the denominator is non-zero. ${ }^{2}$

\footnotetext{
${ }^{2}$ The denominator is zero if and only if $S$ is a constant, which implies that the introduction of a CCP has no effect on the variance of netted exposures.
} 


\subsubsection{Effect of changing the number of asset classes $K$}

Note that the left-hand sides of both expressions $(\star)$ and $(\dagger)$ are increasing in $K$. This means that, as the number of asset classes increases, the introduction of a CCP delivers fewer netting benefits. The intuition here is that, as $K$ increases, the CCP clears a lower proportion of the dealers' activity with one another, so the benefit of netting with the $\mathrm{CCP}$ is reduced. This is consistent with the findings of Duffie and Zhu.

For a given network structure, we can define $K^{*}$ as the critical value of $K$ below which expected net exposures decrease upon introduction of a CCP, and above which they increase. $K^{\dagger}$ is the corresponding critical value for variance. Then:

$$
\begin{gathered}
K^{*} \equiv \frac{1}{4}\left(\frac{\mathrm{E}[S]}{\mathrm{E}[\sqrt{S}]}+\frac{\mathrm{E}[\sqrt{S}]}{\mathrm{E}[S]}\right)^{2} \\
K^{\dagger} \equiv \frac{1}{4}\left(\frac{\operatorname{Var}[S]-\operatorname{Var}[\sqrt{S}]}{\mathrm{E}\left[S^{\frac{3}{2}}\right]-\mathrm{E}[S] \mathrm{E}[\sqrt{S}]}\right)^{2}+1
\end{gathered}
$$

In other words, expressions $(\star)$ and $(\dagger)$ are equivalent to, respectively, $K<K^{*}$ and $K<K^{\dagger}$. These expressions are the focus of the analysis moving forward. They relate the impact of introducing a CCP to the number of asset classes $K$ on the left-hand side, and to the degree distribution $S$ on the right-hand side.

\section{Examination of different network structures}

In this section we consider how the impact on dealer agents of introducing a CCP is affected by the underlying structure of the network. The structure of the network is reflected on the right-hand sides of expressions for $K^{*}$ and $K^{\dagger}$ via the distribution of the random variable $S$.

As a network is a very general object, we restrict our analysis to the most interesting cases. First we apply our results to Duffie and Zhu's network and recover their results. Then we apply our analysis to core-periphery and fat-tailed networks, in order to examine the effect on the results of the presence of large well-connected counterparties, which are typically present in real-world financial networks.

\subsection{Homogeneous network of Duffie and Zhu}

Duffie and Zhu (2011) assume the network is completely connected, so every agent has full degree. This means that $S=N-1$ with certainty, and so $K^{*}=\frac{N^{2}}{4(N-1)}$, which corresponds to their equation 6.

Duffie and Zhu do not consider the effect of the CCP on variance of netting exposures. For their network, both sides of (13) evaluate to zero, so introducing a CCP has no effect on variance. This means that the benefit of the CCP can be considered just by examining the effect on the mean. This is true in general for any network where $S$ is a constant. 
A slight generalization of Duffie and Zhu's completely connected network is the Erdős-Rényi random network, where links are formed independently with some probability $p$. In this network, the nodes are still homogeneous but there may be random variations in their link patterns. As an Erdős-Rényi network grows in size, $K^{*}$ and $K^{\dagger}$ grow without limit (this is a consequence of a Proposition which we prove in section 4.3.2). However, the Erdős-Rényi homogeneous network is not the focus of our paper, as it is not a good fit to real-world financial networks (as shown, for example, in Markose (2012) and Craig and von Peter (2014)). Instead we focus on two models which have been shown to be more realistic: scale-free and core-periphery network structures.

\subsection{Fat-tailed and scale-free networks}

Many real-world financial networks have degree distributions with significant excess kurtosis. There is likely to be a small number of highly-connected nodes (we can think of these as the major dealers), with the majority of nodes having few connections. Fattailed networks can be defined as those whose degree distribution has a tail which can be expressed as $\mathrm{P}(S=s) \sim s^{-\alpha}$, for some real-valued parameter $\alpha>1$. Fatter tails are associated with lower values of $\alpha$.

In this section we will focus on scale-free networks, which are a particular class of fat-tailed networks that have been shown to arise in many real-world applications, including financial networks. ${ }^{3}$ Focusing on this class is instructive because they arise according to a simple and intuitive process, as explained in the following section.

\subsubsection{How do scale-free networks arise?}

Barabási and Albert (1999) show that scale-free networks can be formed via growth and preferential attachment. As time goes on, new nodes join the network and tend to form links with the nodes which are better-connected. This is a realistic model of a derivatives trading network. Over time we would expect new dealers to enter as the market grows. And there are several reasons why these dealers are more likely to trade with the agents which are already better-connected: for example name recognition, an existing relationship in another market or economies of scale may mean that the better-connected agents can offer more attractive terms. Barabási and Albert show that networks formed through this process have fat tails with exponent $\alpha=3$.

For our analysis, we need to settle on a specific form of Barabási-Albert network. We use the scale-free network formation process described in Dorogovtsev, Mendes and Samukhin (2001); henceforth we refer to this as the DMS network. The major advantage of the DMS network is that it has an exact solution for a network of any size. In contrast, Barabási and Albert's general solution only applies when the number of nodes becomes very large, and the assumption of a large network is not necessarily realistic for our purposes. For example Duffie and Zhu consider a network of size 12, which is the number of entities that, at the time of writing their paper, had partnered

\footnotetext{
${ }^{3}$ See, for example, Soramäki et al (2007), Inaoka et al (2004) and Garlaschelli et al (2005).
} 
with ICE Trust to create a CCP for clearing credit default swaps. ${ }^{4}$ The DMS network generating process is as follows:

1. Begin at time $t=2$ with 3 nodes. Each has two links connecting to one another.

2. At each time step, randomly choose an existing link (each with equal probability). A new node is added with two links, which are connected to the two nodes which share the randomly-chosen existing link. Repeat.

3. This process generates an undirected DMS network. We now need to determine the value of each link. As in the analysis of homogeneous networks, we assume that if two dealers have a link in one asset class, then they have a link in all $K$ asset classes. For each pair of connected nodes $i$ and $j$, we generate the net exposures $X_{i j}^{k}, k=1, \ldots, K$, as $K$ iid normal random variables with mean 0 and standard deviation $\sigma$.

This produces a network of size $N=t+1$, which tends towards a scale-free network with exponent $\alpha=3$ as $t$ becomes large. The left-hand panel of Figure 1 shows the realization of a DMS process for $t=100$.

Figure 1: Left-hand panel: Scale-free network of size $t=100$, generated using the DMS process. Right-hand panel: Core-periphery network of size $N=100$, generated using a Bernouilli distribution with parameters $z=0.25, p=0.2, c_{0}=0$.

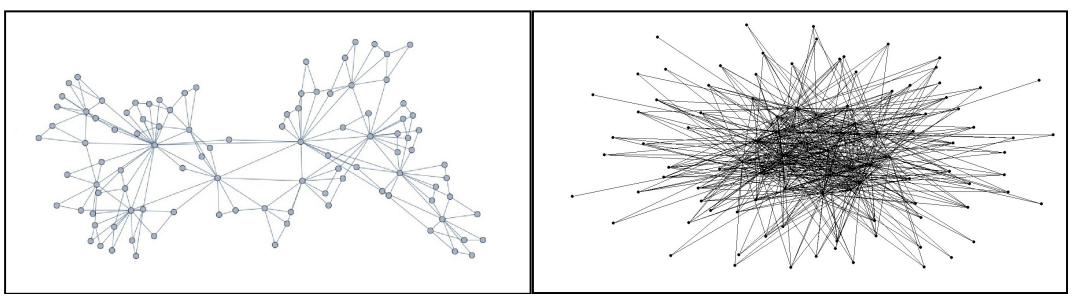

\subsubsection{Asymptotic analysis of fat-tailed and scale-free networks}

We can use the definition of fat tails to approximate the moments of the degree distribution as the size of the network $N \rightarrow \infty$ :

$$
\mathrm{E}\left[S^{m}\right] \sim \int_{Z}^{N-1} s^{m-\alpha} d s
$$

where $Z$ is some constant.

Thus, as $N \rightarrow \infty$ :

$$
\mathrm{E}\left[S^{m}\right] \sim \begin{cases}O\left((N-1)^{m-\alpha+1}\right) & \text { if } m>\alpha-1 \\ \log (N-1) & \text { if } m=\alpha-1 \\ O(1) & \text { if } m<\alpha-1\end{cases}
$$

\footnotetext{
${ }^{4}$ Sizes of other real-world CCP networks can be found in Table 1 in Galbiati and Soramäki (2012) and footnote 12 in Cox, Garvin and Kelly (2013).
} 
and so $\mathrm{E}\left[S^{m}\right]$ has a finite limit if and only if $m<\alpha-1$.

We can apply this result to our expressions for $K^{*}$ and $K^{\dagger}$. The table below summarizes the asymptotics for various values of $\alpha$.

Table 1: Asymptotic behavior of $K^{*}$ and $K^{\dagger}$ in a fat-tailed network.

\begin{tabular}{c|cc}
\hline \hline & $K^{*}$ & $K^{\dagger}$ \\
\hline $1<\alpha \leq 2$ & tends to positive infinity & tends to positive infinity \\
$2<\alpha \leq 3$ & tends to finite limit & tends to positive infinity \\
$\alpha>3$ & tends to finite limit & tends to finite limit \\
\hline
\end{tabular}

When $\alpha>3$, the right-hand sides of both expressions tend to finite limits. This means that there will be some critical values $K^{*}$ and $K^{\dagger}$ beyond which the introduction of a CCP will increase the expectation and variance of net exposures no matter how large the network is. But when $1<\alpha \leq 2$ the converse is true: for any given number of asset classes $K$, there will be some critical network size beyond which the expectation and variance of net exposures decline with the introduction of a CCP. This suggests that a CCP is more likely to deliver benefits to dealers in networks with fatter tails. This makes intuitive sense, because networks where the major dealers are highly important are more likely to benefit from bilateral netting with these dealers rather than with a $\mathrm{CCP}$ which clears only a single asset class.

For real-world networks $\alpha$ typically lies between 2 and 3. This is the intermediate case in the table, and so there may conceivably be a trade-off between a higher expected value of net exposures and a lower variance. In particular, if the number of asset classes is larger than the asymptotic limit of $K^{*}$, then we will certainly see such a trade-off. This is clearly the case for scale-free networks, which have $\alpha=3$.

\section{A lemma for the asymptotic DMS network}

Lemma: For the infinite DMS network, novating a single asset class to a CCP is never beneficial in terms of netting efficiency, regardless of the number of asset classes $K$ (except in the trivial case $K=1$ ). But is always beneficial in terms of the variance of net exposures.

Proof: We have already shown the second part is true, because $\alpha=3$ for the DMS network and so $K^{\dagger}$ grows without bound.

Now we just need to show that the asymptotic value of $K^{*}$ - which we know tends to a finite limit - is smaller than 2. Observe that $\mathrm{E}_{t}(S)=\frac{4 t-2}{t+1} \rightarrow 4$ in the DMS network, since at each step of the network construction process four additional links are created (each new node has an in-link and an out-link with two existing nodes). Dorogovstev, Mendes and Samukhin (2001) show that:

$$
\lim _{t \rightarrow \infty} \mathrm{P}_{t}(s)=\frac{12}{s(s+1)(s+2)},
$$


and so the term $\frac{\mathrm{E}_{t}[S]}{\mathrm{E}_{t}[\sqrt{S}]}$ is asymptotically equal to:

$$
4 /\left(12 \sum_{s=2}^{\infty} \frac{1}{\sqrt{s}(s+1)(s+2)}\right)=2.17
$$

This gives us an asymptotic value for $K^{*}=1.73<2$. Therefore in the infinite limit the CCP never improves expected netting efficiency, except in the trivial case where $K=1$; i.e. when the CCP clears the only asset class.

Note, however, that while the increase in expected exposures that results from the introduction of a CCP $\left(\widetilde{\phi_{N, K}}-\phi_{N, K}\right)$ is finite, the reduction in variance $\left(\widetilde{v_{N, K}}-v_{N, K}\right)$ is without bound. This means that, so long as agents' preference functions put any weight on volatility - i.e. there is at least some degree of risk aversion — then, for any given $K$, then there must be some critical size of the network above which introducing a $\mathrm{CCP}$ is beneficial for the dealer agents.

\subsubsection{Finite analysis of the scale-free network}

The distribution of $S$ for a given $t$ is as given in Dorogovtsev, Mendes and Samukhin (see their equation 8 ):

$$
\mathrm{P}_{t}(s)=\frac{t}{t+1}\left[\frac{s-1}{2 t-3} \mathrm{P}_{t-1}(s-1)+\left(1-\frac{s}{2 t-3}\right) \mathrm{P}_{t-1}(s)\right]+\frac{1}{t+1} 1_{[s=2]}
$$

for $t \geq 3$, with initial condition $\mathrm{P}_{2}(s)=1_{[s=2]}$. (Here, $1_{[\cdot]}$ denotes the identity function which takes the value 1 if the condition in the subscript is true, and zero otherwise.)

Figure 2 shows the effect of introducing a CCP for a range of values of $(K, t)$. The frontier of the black area is equal to $K^{*}$, while the frontier of the gray area is equal to $K^{\dagger}$. The distribution of $S$ is determined by equation (20) for that value of $t$. This has been calculated for DMS networks with up to 500 members.

$K^{*}$ tends monotonically upward to the value 1.73 , which accords with the asymptotic limit identified in the subsection above. This confirms that the introduction of a CCP will increase net exposures for any non-trivial DMS network (i.e. one where $K \geq 2$ ). In contrast, $K^{\dagger}$ (shown by the frontier of the gray area) increases without limit as $\sim O\left(\log (N-1)^{2}\right)$, as predicted by the asymptotic analysis. For sufficiently large networks the introduction of a CCP will cause a reduction in the variance of exposures.

\subsection{Core-periphery networks}

Core-periphery networks have been presented in the recent literature as an alternative to scale-free networks as a model of real-world financial linkages. These networks are characterized by a partition of the nodes into two sets: a heavily-connected set of 'core' nodes, along with a sparsely connected set of 'peripheral' nodes. In most models this partition is determined exogenously.

Borgatti and Everett (1999) present a general model to allow for the detection of core-periphery networks: they assume that in such networks all core nodes are linked 
Figure 2: The effect of the introduction of a CCP in a DMS scale-free network, for a range of values of $(K, t)$. The chart is calculated up to $t=499$ - that is, $N=500$. Values of $K^{*}$ are represented by the frontier between the black and gray areas. Values of $K^{\dagger}$ are represented by the frontier between the gray and white areas.

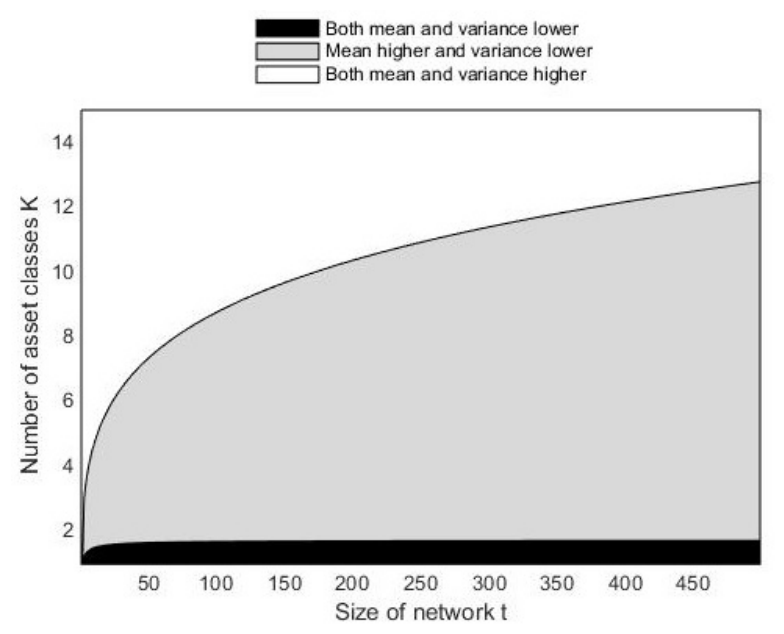

to one another, while there are no links between peripheral nodes. They then present a statistic to test for correlations between such an idealized core-periphery network and the actual data. Their model is agnostic about the distribution of links between core and periphery nodes; this is because their specification is aimed at empirical verification of the structure, rather than a general model of a core-periphery network.

Langfield, Liu and Ota (2014) and Craig and von Peter (2014) use the BorgattiEverett approach to identify core-periphery structures in the UK and German interbank markets respectively. Wetherilt, Zimmerman and Soramäki (2010) are able to describe changes in the UK overnight money market using a more generalized maximum likelihood approach, which is possible because of the small size of their network. And Markose (2012) characterizes the global network of OTC derivatives as a coreperiphery network, but does not test this against other possible structures.

\subsubsection{How do core-periphery networks arise?}

Van der Leij, in't Veld and Hommes (2014) show that core-periphery structures can arise as the stable outcome of a process of strategic network formation between heterogeneous agents. In their model, there are 'big' banks and 'small' banks, and the payoff from forming a link with a big bank is greater than a link with a small bank.

Abstracting away from consideration of individual nodes' optimal strategies, we can characterize the formation of a core-periphery network using the following simple process: 
1. Begin with $c_{0}$ core nodes, which are connected to each other.

2. At each step a new node is added. With probability $z$ this new node is labeled 'core'. Otherwise, the node is labeled 'peripheral'.

3. A new core node forms links with all of the existing core nodes with certainty, and also forms links with each of the existing peripheral nodes according to some given distribution $h$, which may depend on the link structure of the network at that point in time. A new peripheral node never forms links with existing peripheral nodes, but will form links with existing core nodes according to the given distribution $h$.

This process, repeated $N-c_{0}$ times, will produce a network of size $N$ which meets the Borgatti-Everett definition of a core-periphery network. The parameters of the model are $c_{0}, z$ and the distribution $h$. Borgatti and Everett allow any feasible distribution to determine core-periphery links; for example $h$ could depend on existing links in the network. But the most natural and simple way to model links between the core and periphery is to assume that each link occurs independently with some fixed probability $p \in(0,1)$ - that is, the link formation process follows a Bernouilli distribution. Under this assumption, the number of links for any randomly chosen node can be expressed as a mix of binomial distributions plus a constant. ${ }^{5}$ The right-hand panel of Figure 1 shows a realization of such a network-generating process. We will focus on the Bernouilli core-periphery network later in this section.

Underlying this network generation process is the assumption that links to core nodes are desirable, while links to peripheral nodes are not. There are plausible reasons why this may be the case for real-world financial networks. Agents may prefer to deal with larger players who they are more likely to have existing relationships with in other markets. Exposures to larger players may be easier to monitor. And economies of scale may mean that these larger players offer more attractive trading terms.

\subsubsection{Asymptotic analysis of core-periphery networks}

In order to make asymptotic inferences about the Bernouilli core-periphery network, we will state and prove a more general Proposition.

\section{A proposition on the asymptotic limit of networks with 'thin tails'}

Proposition: Suppose the degree distribution $S$ of the network has the following properties:

- $\mathrm{E}[S] \rightarrow \infty$ as $N \rightarrow \infty$;

- $\operatorname{Var}[S] / \mathrm{E}[S]^{2}$ tends to a finite limit as $N \rightarrow \infty$;

- Higher moments tend to zero as $N \rightarrow \infty$.

\footnotetext{
${ }^{5}$ Let $C$ be the number of core nodes. Then $C \sim c_{0}+\operatorname{Bin}\left(N-c_{0}, z\right)$, and the conditional distribution of $S$ is $S \mid C \sim z(C-1)+z \cdot \operatorname{Bin}(N-C, p)+(1-z) \cdot \operatorname{Bin}(C, p)$. Note that the cases $p=0$ or $z=1$ correspond to the Duffie and Zhu network with size equal to the core.
} 
Then $K^{*}$ and $K^{\dagger}$ are both $\sim O(\mathrm{E}[S])$ as $N \rightarrow \infty$.

Proof: Let us use $\mu$ and $V$ to denote $\mathrm{E}[S]$ and $\operatorname{Var}[S]$ respectively, for a given $N$. Then, as $N \rightarrow \infty$, we can make use of the following approximations:

$$
\mathrm{E}[\sqrt{S}]=\mu^{0.5} \mathrm{E}\left[1+\left(\frac{S}{\mu}-1\right)\right]^{0.5} \approx \mu^{0.5}\left[1-\frac{V}{8 \mu^{2}}\right]
$$

and

$$
\mathrm{E}\left[S^{\frac{3}{2}}\right]=\mu^{1.5} \mathrm{E}\left[1+\left(\frac{S}{\mu}-1\right)\right]^{1.5} \approx \mu^{1.5}\left[1+\frac{3 V}{8 \mu^{2}}\right] .
$$

In both cases we have expanded the binomial series around 1 and neglected cubic and higher order terms. Substituting these into our expressions for $K^{*}$ and $K^{\dagger}$ and using the properties of $S$, we find that both $K^{*}$ and $K^{\dagger} \sim O(\mu)$.

For a network which meets the conditions in the Proposition, $K^{*}$ and $K^{\dagger}$ will increase without bound as the network becomes large. This suggests that, for any given number of asset classes $K$, there is a minimum size of the network above which the introduction of a CCP would reduce both mean and variance. This is consistent with the findings of Duffie and Zhu, where a larger network tends to make a CCP more likely to be beneficial.

Examples of networks which meet these 'thin-tailed' conditions are the ErdôsRényi described in section 4.1, and the Bernouilli core-periphery network described above. ${ }^{6}$ In both cases, $\mathrm{E}[S] \sim N-1$.

This proposition does not apply to a network with a link formation process $h$ which generates fat tails. For example, the case where core and peripheral nodes form links according to a Barabási-Albert preferential attachment process would be likely to generate asymptotic results similar to those derived in section 4.2.2. In particular, Markose (2012) shows that the network of global OTC derivatives exposures can be modeled by a core-periphery network with fat tails in the degree distribution. In this case, the fattailed network analytics would be more appropriate when considering central clearing.

\subsubsection{Finite analysis of the Bernouilli core-periphery network}

While the only parameter in the DMS network is its size $N=t+1$, the Bernouilli core-periphery network has three additional parameters $z, p$ and $c_{0}$. In order to carry out numerical solutions, we need to choose feasible parameter values, so we turn to the empirical literature. The table below summarizes parameter estimates from three selected papers: the global OTC derivatives network from Markose (2012), the Dutch interbank market from Van der Leij et al (2014), and the German interbank market from Craig and von Peter (2014). ${ }^{7}$ The value of $c_{0}$ is impossible to observe and is likely to make little difference to larger networks, so we assume $c_{0}=0$.

\footnotetext{
${ }^{6}$ As the number of trials of a binomial distribution tends to infinity, its excess kurtosis and skewness both tend to zero.

${ }^{7}$ For the global OTC network we use the 'inner core' as defined by Markose. Each paper provides the number of nodes $N$, the size of the core $C$ and the total number of directed links $L$. We assume that $c_{0}=0$. We can then estimate $z=\frac{C}{N}$ and $p=\frac{L-C(C-1)}{2 C(N-C)}$.
} 
Table 2: Parameter estimates for core-periphery networks in the literature.

\begin{tabular}{l|lll}
\hline \hline & Global OTC & Dutch interbank & German interbank \\
\hline Period & 2009 Q4 & 2008 Q4 & 2003 Q2 \\
$N$ & 204 & 100 & 1802 \\
Estimated $z$ & 0.10 & 0.15 & 0.02 \\
Estimated $p$ & 0.01 & 0.31 & 0.11 \\
\hline
\end{tabular}

These three networks have $z$ and $p$ fairly small, as might be expected. We will use $z=0.10, p=0.10$ for our numerical solutions - these parameter values are fairly representative of Table $2 .{ }^{8}$

Figure 3 shows the effect of introducing a CCP for various values of $N$ up to 500, for the parameters $z=0.10, p=0.10, c_{0}=0$. As predicted, $K^{*}$ and $K^{\dagger}$ increase approximately linearly with $N$. As with scale-free networks, there is a gray intermediate area where $K^{*}<K<K^{\dagger}$ - in such cases, introducing a CCP will increase expected net exposures in the network, but reduce the variance. Further numerical simulations of these networks with other parameter values indicate that $K^{\dagger}$ is larger than $K^{*}$ and increases more rapidly with $N$, suggesting that the gray intermediate areas always exist.

Figure 3: The effect of the introduction of a CCP in a core-periphery network with $z=0.10, p=0.10, c_{0}=0$, for a range of values of $(K, N)$ up to $N=500$. Values of $K^{*}$ are represented by the frontier between the black and gray areas. Values of $K^{\dagger}$ are represented by the frontier between the gray and white areas.

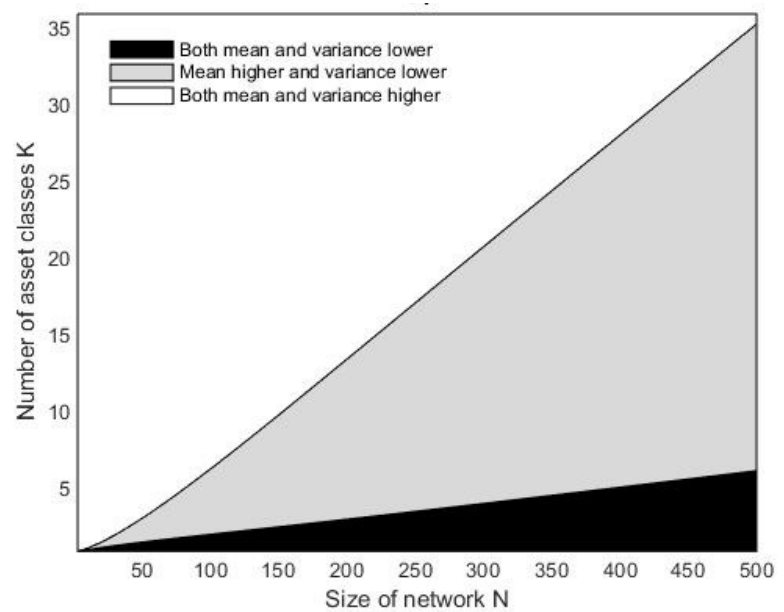

\footnotetext{
${ }^{8}$ The Markose parameter values may be thought to be the best proxy, given that we are interested in central clearing of OTC derivatives and not other forms of interbank lending. However, it should be noted that the Markose network exhibits fat tails and so does not conform very well to a Bernouilli core-periphery structure.
} 
As Figure 3 shows, there are network sizes $N$ where a CCP is unambiguously bad for netting efficiency. For $N \leq 93, K^{*}<2$ and so a CCP will never reduce average net exposures for networks of this size. And for $N \leq 29, K^{\dagger}<2$ and so introducing a CCP for networks of this size increases both the mean and variance of net exposures.

Testing other parameter values, we also find that $K^{*}$ and $K^{\dagger}$ always increase with $N$ and decrease with both $z$ and $p$. And, comparing Figure 3 with Figure 2, we can see that for a given number of dealer agents, the introduction of a CCP is more likely to deliver benefits in a core-periphery network than in a scale-free network. Both of these results arise because, when the network relies on links with a small number of key nodes, bilateral netting substantively reduces net exposures, and central clearing disrupts this. But when links are more spread amongst a larger number of key nodes, central clearing may bring greater netting benefits. ${ }^{9}$

This strongly suggests that knowledge of network structure - as well as the size and the number of asset classes - is important in assessing the possible benefits of central clearing on netting efficiency. Particularly important is the degree structure of the largest nodes in the network.

\section{Concluding remarks}

We extend the analysis of Duffie and Zhu (2011) to a wider variety of networks and show that the novation of a single asset class to a CCP will materially affect both the mean and variance of net exposures. Both mean and variance will tend to decrease if the network is larger or if there are a smaller number of asset classes traded in the network. But central clearing brings fewer netting benefits if the network relies on a small number of key nodes for most of its links.

In the case that the structure of the trading network is scale-free or in the growth process of a scale-free network, introducing a CCP will always increase expected net counterparty exposures, unless the CCP clears all asset classes. This is also true for smaller core-periphery networks. But this increase in expected net exposures may be accompanied by a reduction in the volatility of these exposures. This is more likely for larger networks and those which trade and net a smaller number of asset classes.

This has welfare implications, because net exposures relate to aggregate counterparty risk in the network, and to margin needs. If central clearing reduces both the expectation and variance of exposures, it would appear to be beneficial. If central clearing increase both the expectation and variance, then it would appear to reduce welfare. But in many cases the expectation increases while the variance falls (shown by the gray areas on our charts). In such cases, whether central clearing improves welfare or not here will depend on the risk appetite of the dealers in the network, policymakers and any other relevant agents. In general, if decision-makers are more risk averse, then they are likely to place more value on the effect on variance than the effect on mean, and so are more likely to favor the introduction of a CCP.

\footnotetext{
${ }^{9}$ This is supported by Table 1 which shows that, as $\alpha$ increases in a fat-tailed network, central clearing tends to bring netting benefits. That is because fatter tails means a greater number of nodes with a substantial number of links.
} 
In our model, it appears that the benefit of central clearing accrues to the dealer agents. The question may then arise, what is the justification for regulatory intervention? In other words, what market failure does a regulator address by mandating central clearing, given that the dealer agents have chosen not to set up a CCP themselves?

One likely answer is that the dealers are less risk-averse than is socially optimal. This may be the case, for example, if high or volatile margin requirements impose externalities on markets for the collateral assets (Murphy, Vasios and Vause (2014)), or if agency problems mean that dealers take excessive risks. In such cases, a systemic risk regulator should have a higher degree of risk aversion than the dealer agents, and so may determine that the optimal policy measure is to mandate central clearing.

Another plausible explanation is that, while a CCP may improve netting efficiency in aggregate, it does not provide an improvement for every party in the network. ${ }^{10} \mathrm{In}$ other words, it is socially efficient but not Pareto-efficient. This may make it difficult for the agents in the network to agree to set up a CCP themselves. Even if they are able to do so, the agents who do not benefit from central clearing would find it optimal to continue to trade bilaterally, which would disrupt netting sets for those who do use the CCP and make it less effective. This provides a reason for a regulator to intervene and mandate central clearing, in order to make the market as a whole more efficient.

As in Duffie and Zhu we do not account for the fact that exposures to the CCP are likely to carry much less risk than those to commercial trading counterparties. One key purpose of a CCP is to reduce counterparty risk. Once risk-weights are taken into account, total expected risk in the network may be reduced, further increasing the benefits of the CCP. ${ }^{11}$ Furthermore, once a CCP is introduced the participants in the network may find it optimal to adjust their exposures to one another. As in Duffie and Zhu, we do not attempt to model this endogeneity.

We also do not assume any correlation between magnitudes of exposures in different asset classes. In the event that two asset classes tend to have positively correlated exposures, bilateral netting between these assets will have limited benefits and so a CCP which clears exactly one of these assets may improve netting efficiency. Similarly, we assume that a link between two agents in one asset class implies a link in all asset classes. Although we argue this may be a realistic assumption, in a network with greater specialization there will be fewer opportunities for bilateral netting between counterparties, so a CCP would be likely to deliver greater benefits.

In this paper we have adopted a theoretical approach to the problem, as we have assumed that only the distribution of the network and exposures are known, and not the exact network structure. If the empirical structure is known, then the empirical distribution $S$ can be plugged into our equations. As part of the regulatory reforms following the financial crisis, regulators have increasingly better access to OTC derivatives data. However, in general these data will not be back-dated - in particular, regulators may not have data on network structures leading into the last crisis - so it may not be possible to observe the empirical network structure at precisely the point when risk-aversion is at its peak. It may also be difficult for regulators to piece together exposure data

\footnotetext{
${ }^{10}$ Heath, Kelly and Manning (2013) find that the netting benefits of central clearing accrue disproportionately to core nodes.

${ }^{11}$ Anderson, Dion and Pérez Saiz (2013) adopt this risk-weighting approach.
} 
about different asset classes and identify different corporate entities as part of the same dealer agent. Our approach allows a regulator not to be concerned about the identity of each node, but to use the structure of the network as a whole.

Moreover, networks develop and grow over time and so historical data may not be useful for understanding an innovative or growing market. As the network structure rapidly changes in real time, regulators may find it more useful to model the growth dynamics rather than rely on observed data which rapidly becomes stale. Our model allows for this.

This paper focuses on central clearing, but our model can be applied to any network where the issue of bilateral vs centralized netting is under consideration. For example, it could be used to consider the effect multilateral netting in the interbank market, the benefits of bilateral vs triparty repo, or the effect on payment system exposures of introducing a liquidity-saving mechanism.

\section{References}

Anderson S, Dion, J-P and Pérez Saiz, H (2013), “To link or not to link? Netting and exposures between central counterparties", Journal of Financial Market Infrastructures, Vol. 1, No. 4, pages 3-29.

Barabási, A L and Albert, R (1999), "Emergence of scaling in random networks", Science, Vol. 286, pages 509-512.

Borgatti, S P and Everett, M G (1999), "Models of core/periphery structures", Social Networks, No. 21, pages 375-395.

Borovkova, S and Lalaoui El Mouttalibi, H (2013), "Systemic risk and centralized clearing of OTC derivatives: a network approach", Social Science Research Network.

Campbell, S (2014), "Estimating the effect of central clearing on credit derivative exposures", FEDS Notes, February 26.

Cont, R and Kokholm, T (2014), "Central clearing of OTC derivatives: bilateral vs multilateral netting”, Statistics and Risk Modeling, Vol. 31, No. 1, pages 3-22.

Cox, N, Garvin, N and Kelly, G (2013), "Central counterparty links and clearing system exposures", Reserve Bank of Australia Research Discussion Paper, 2013-12.

Craig, B and von Peter, G (2014), "Interbank tiering and money center banks", Journal of Financial Intermediation, Vol. 23, pages 322-47.

Dorogovtsev, S N, Mendes, J F F and Samukhin, A N (2001), "Generic scale of the 'scale-free' growing networks", Physical Review E, 63(062101).

Duffie, D, Scheicher, M and Vuillemey, G (2014), "Central clearing and collateral demand", European Central Bank Working Paper, No. 1638.

Duffie, D and Zhu, H (2011), "Does a central clearing counterparty reduce counterparty risk?", Review of Asset Pricing Studies, Vol. 1, No. 1, pages 74-95.

Galbiati, M and Soramäki, K (2012), "Clearing networks", Journal of Economic Behavior \& Organization, Vol. 83, pages 609-626.

Garlaschelli, D, Battiston, S, Castri, M, Servedio, V D P and Caldarelli, G (2005), "The scale-free topology of market investments", Physica A, No. 350, pages 491-99.

Heath, A, Kelly, G and Manning (2013), "OTC derivatives reform: netting and networks", Liquidity and Funding Markets, Conference Proceedings, August 2013. 
Inaoka, H, Ninomiya, T, Shimizu, T, Takayasu, H and Taniguchi, K (2004), "Fractal network derived from banking transaction - an analysis of network structures formed by financial institutions", Bank of Japan Working Paper, No. 04-E-04.

Jackson, J P and Manning, M J (2007), "Comparing the pre-settlement risk implications of alternative clearing arrangements", Bank of England Working Paper, No. 321.

Langfield, S, Liu, Z and Ota, T (2014), "Mapping the UK interbank system", Journal of Banking \& Finance, Vol. 45, pages 288-303.

Markose, S (2012), "Systemic risk from global financial derivatives: a network analysis of contagion and its mitigation with super-spreader tax", IMF Working Paper, No. $12 / 282$.

Murphy, D, Vasios, M and Vause, $\mathbf{N}$ (2014), 'An investigation into the procyclicality of risk-based initial margin models", Bank of England Financial Stability Paper, No. 29.

Sidanius, C and Žikeš, F (2012), "OTC derivatives reform and collateral demand impact", Bank of England Financial Stability Paper, No. 18.

Song, R, Sowers, R B and Jones, J (2014), "The topology of central counterparty clearing networks and network stability", Stochastic Models, Vol. 30, No. 1, pages 16-47.

Soramäki, K, Bech, M L, Arnold, J, Glass, R J and Beyeler, W E (2007), "The topology of interbank payment flows", Physica A, Vol. 379, pages 317-33.

Van der Leij, M, in't Veld, D and Hommes, C (2014), "The formation of a core periphery structure in financial networks", Tinbergen Institute Discussion Paper, 14098/II.

Wetherilt, A, Zimmerman, P and Soramäki, K (2010), “The sterling unsecured loan market during 2006-08: insights from network theory”, Bank of England Working Paper, No. 398. 